\title{
Critical structure of the QCD medium
}

\author{
Bernd-Jochen Schaefer* \\ Institut für Physik, Karl-Franzens-Universität, A-8010 Graz, Austria \\ E-mail: bernd-jochen.schaefer@uni-graz.at
}

Fluctuations in the vicinity of a phase transition are important but neglected in mean-field theory. In order to assess the influence of such fluctuations on the critical endpoint and the size of the critical region in the QCD phase diagram, a mean-field calculation of a two-flavor quark-meson model is compared with a renormalization group approach. However, due to the lack of confinement in this effective model the equation of state near the chiral phase transition is still unrealistic. A first improvement of this model can be achieved by coupling quark degrees of freedom to the Polyakov loop, consequently incorporating certain aspects of confinement. The influence of these modifications on the resulting phase diagram is discussed.

Critical Point and Onset of Deconfinement 4th International Workshop

July 9-132007

GSI Darmstadt,Germany

${ }^{*}$ Speaker. 


\section{Introduction}

Driven by the future heavy-ion programs of CBM at the FAIR facility, and soon also at the CERN LHC, the properties of strongly interacting matter at finite temperature $T$ and baryon number density are of growing interest. On the theoretical side QCD at high temperature and/or quark chemical potential $\mu$ predicts a phase transition from the ordinary hadronic phase to a chirally restored and deconfined quark gluon plasma (QGP) phase. Based on calculations in effective models, as well as universality arguments, the order of the phase transition depends on the number of quark flavors and on the value of the quark masses. For physical values of the quark masses and small baryon densities the transition as function of temperature is continuous. On the other hand, for small temperature the transition as function of the chemical potential is of first-order. This suggests the existence of a critical endpoint (CEP) in the phase diagram, given by the endpoint of a first-order transition line in the $(T, \mu)$-plane $[1,2,3]$. The phase transition at the CEP is of second-order and belongs to the three-dimensional Ising universality class. At this point the QCD free energy has a genuine singularity and as a consequence both the chiral and the quark number susceptibility diverge. Due to, e.g., the large strange quark-mass sensitivity the precise location of this endpoint is not yet known.

From QCD lattice simulations for vanishing $\mu$, it is known that the transition temperature in the presence of light quarks is lowered substantially from its value in the pure gauge limit of infinitely heavy quarks. Furthermore, in the limit of vanishing up- and down quark masses as well as infinite strange quark mass, the chiral transition is likely to be of second-order and its static critical behavior falls in the universality class of the Heisenberg $O(4)$ model in three dimensions [4]. But the situation on the lattice for finite $\mu$ is much less clear due to the notorious fermion sign problem. Nevertheless, from direct numerical evaluations of the QCD partition function or also from a Taylor expansion of the pressure around vanishing $\mu$ some evidence exists for a CEP in the phase diagram at finite $\mu[5,6,7]$. For two-flavor massless QCD the CEP turns into a tricritical point (TCP) where for small $\mu$ 's the $O(4)$ line of critical points terminates. For larger $\mu$ 's the transition is again of first-order.

In order to interpret the physical content of these lattice findings they have to be compared to model studies. A variety of model studies of the CEP and the critical region are available in the literature, but most of them are based on a mean-field description of the phase transition, consequently neglecting quantum fluctuations. However, it is well-known, especially in the vicinity of a phase transition, that fluctuations become more and more important and mean-field theory fails to describe adequately the critical behavior of phase transitions. Therefore, it is necessary to go beyond mean-field theory to arrive at a proper description. An efficient way, to go beyond mean-field theory, is the renormalization group (RG) method which considers the universal and non-universal aspects not only of second-order but also of first-order phase transitions $[8,9]$.

In this contribution we summarize recent results on the size of the critical region around the TCP and CEP obtained with an effective two-flavor quark-meson model, both for a mean-field approximation and a RG approach [10]. Due to the lack of confinement in this model single quark states are already excited in the chirally broken phase yielding an unrealistic equation of state (EoS) near the phase transition [11, 12]. Coupling quark degrees of freedom to the Polyakov loop certain aspects of confinement are incorporated that improve the EoS [13, 14, 15, 16]. Finally, the 
modifications caused by the Polyakov loop on the phase diagram is presented and compared with the one obtained in the pure quark-meson model [17].

\section{The size of the critical region around the TCP and CEP}

The size of a critical region around a critical endpoint is defined through the breakdown of mean-field theory and emergence of nontrivial critical exponents. The size usually can be determined by the well-known Ginzburg criterion which is based on an expansion of the singular part of the free energy for a second-order phase transition. However, since the expansion coefficients are not known for the strong interaction the Ginzburg criterion is only of limited use in the present context. Universality arguments are also not helpful if the underlying microscopic dynamics is not well determined. For example, in the cases of the $\lambda$-transition of liquid $\mathrm{He}^{4}$ and the superconducting transition of metals, both transitions lie in the same universality class but their critical regions defined by their corresponding Ginzburg-Levanyuk temperature deviate from each other by several orders of magnitude.

An estimate for the size of the critical region for hadronic matter also can be defined by calculating the in-medium scalar and chiral static susceptibility using their enhancement as the criterion [18]. In general, static susceptibilities are obtained from the dynamic response function $\chi_{a b}(\omega, \vec{q})$ in the static $(\omega=0)$ and long wavelength limit $(\vec{q} \rightarrow 0)$ where $a, b$ denote some external fields. The scalar static susceptibility $\chi_{\sigma}$ corresponds to the zero-momentum projection of the scalar propagator which encodes all fluctuations of the chiral order parameter $\langle\bar{q} q\rangle$. Thus, the maximum of $\chi_{\sigma}$ as function of temperature or quark chemical potential should coincide with the most rapid change in the chiral order parameter. It is related to the sigma meson mass via $\chi_{\sigma} \sim M_{\sigma}^{-2}$. Similarly, the chiral or quark number susceptibility $\chi_{q}$ is the response of the net quark number density $n_{q}$ to an infinitesimal variation of the quark chemical potential, $\chi_{q}=\partial n_{q} / \partial \mu_{q}$.

In mean-field approximation and for a physical pion mass the two-flavor quark-meson model exhibits a smooth crossover on the temperature axis and a first-order chiral phase transition on the density axis [19]. For increasing temperatures the first-order transition line terminates at the CEP. Along the line of a first-order phase transition the thermodynamic potential has two minima of equal depth which are separated by finite potential barrier. The height of the barrier is largest at zero temperature and finite chemical potential and decreases towards higher temperature. At the CEP the barrier and accordingly the latent heat of the transition disappears and the potential flattens. At this point the phase transition is of second-order and characterized by long-wavelength fluctuations of the order parameter which is in our case proportional to the scalar $\sigma$-field. As a consequence the scalar sigma mass must vanish at this point which can be seen in the behavior of the in-medium meson masses: in the vicinity of the CEP the sigma mass as function of temperature and quark chemical potential drops below the pion mass which stays always finite since the chiral symmetry is still explicitly broken. For temperatures and chemical potentials above the chiral transition the sigma mass increases again and will degenerate with the pion mass signaling restoration of chiral symmetry.

At the CEP the slope of the quark number density tends to infinity which will yield a diverging susceptibility exactly at this point. For temperatures below the critical one the quark number density jumps because of the first-order phase transition. For temperatures above the CEP the discontinuity 


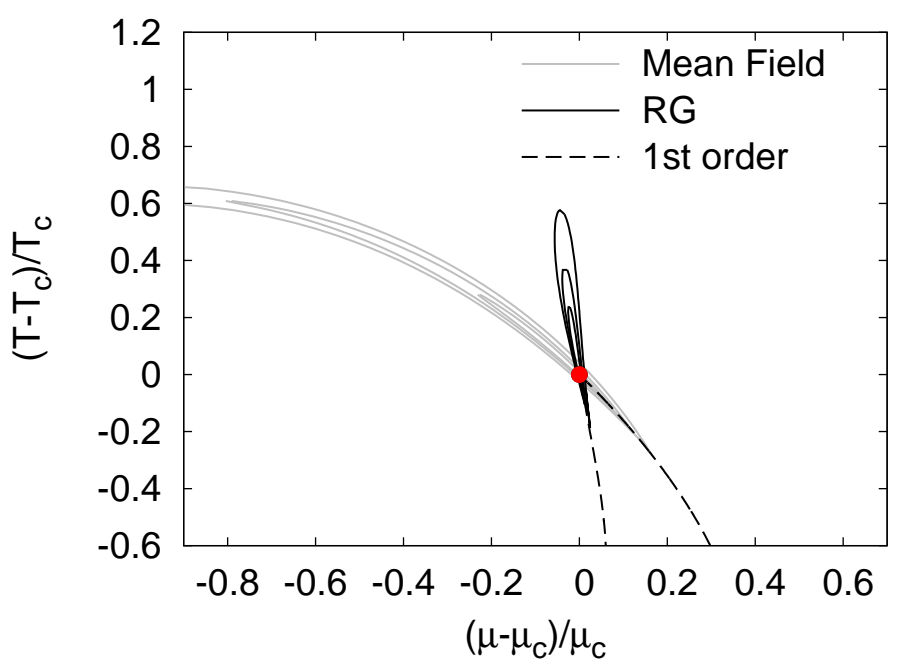

Figure 1: The contour regions in the phase diagram for three different ratios of the scalar susceptibilities $R_{s}=\chi_{\sigma}(T, \mu) / \chi_{\sigma}(0,0)\left(R_{s}=10,15,20\right)$ around the CEP in reduced units.

vanishes across the transition line and the density changes gradually due to the smooth crossover. This finally produces a finite height of the quark number susceptibility $\chi_{q}$. Thus, in equilibrium $\chi_{q}$ diverges only at the CEP and is finite everywhere else ${ }^{1}$. The height of $\chi_{q}$ decreases for decreasing chemical potentials towards the $T$-axis. For temperatures below the CEP $\chi_{q}$ is discontinuous and jumps across the first-order line. In the vicinity of the CEP the quark number density is always finite but the susceptibility becomes large. Since the quark number susceptibility is proportional to the isothermal compressibility $\kappa_{T}$ via the relation $\kappa_{T}=\chi_{q} / n_{q}^{2}$ this behavior indicates that the system is easy to compress around the critical point.

Figure 1 shows a contour plot of the scalar susceptibility divided by the vacuum susceptibility

$$
R_{S}(T, \mu)=\frac{\chi_{\sigma}(T, \mu)}{\chi_{\sigma}(0,0)}
$$

for three fixed ratios around the CEP in the phase diagram in reduced units. The light curves are the mean-field and the other ones the RG results which we will discuss later. The region of the enhanced susceptibility is elongated in the direction of the extrapolated first-order transition line. The deeper reason for this shape can be understood by a study of the critical exponents of the susceptibility which specify its power-law singularity. In the case of the susceptibility the form of this divergence depends on the path by which one approaches the critical point. For the path asymptotically parallel to the first-order transition line the divergence scales with an exponent $\gamma$ which in mean field is $\gamma=1$. For any other path, not parallel to the first-order line, the divergence scales with another exponent $\varepsilon$ which in mean-field theory is equal to $2 / 3$. Since $\gamma>\varepsilon$ the susceptibility is enhanced in the direction parallel to the first-order transition line. This is the reason for the elongated shape of the critical region in the phase diagram.

Universality arguments as well as lattice QCD simulations for two quark flavors without $U_{A}(1)$ anomaly in the chiral limit predict at vanishing quark chemical potential that the effective theory

\footnotetext{
${ }^{1}$ This changes for non-equilibrium systems: when entering the coexisting region of the first-order line, the susceptibility also diverges along the isothermal spinodal line, see [20] for further details.
} 


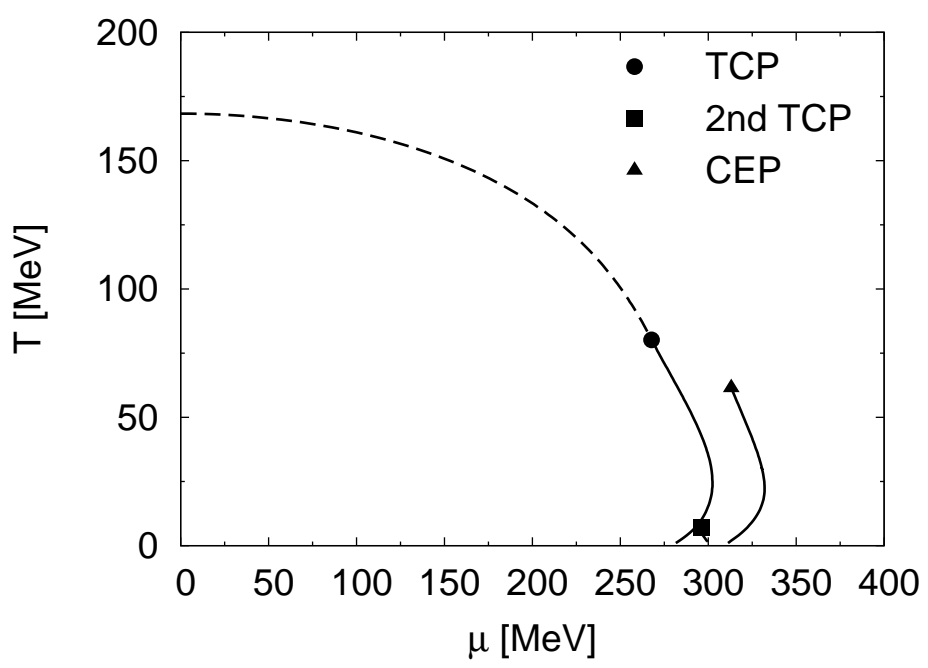

Figure 2: Two phase diagrams for the quark-meson model obtained with the RG: One for physical pion masses (right solid line which ends in the CEP) and another one for the chiral limit. Solid lines denote first-order and dashed lines second-order transition lines.

for the chiral order parameter is the same as for the $O(4)$ model, which has a second-order phase transition. It is expected that the static critical behavior falls into the universality class of the $O(4)$ symmetric Heisenberg model in three dimensions. When reducing the pion mass by varying the explicit chiral symmetry breaking parameter of our quark-meson model, the CEP moves towards the $T$-axis. Already for the pion mass $M_{\pi} \sim 70 \mathrm{MeV}$ the CEP disappears and chiral symmetry is restored via a first-order transition for all temperatures and quark chemical potentials. As a consequence this model does not have a tricritical point in the chiral limit in contradiction to universality arguments and lattice simulations [21]. But as already stated in [19], within the mean-field approximation the order of the phase transition in the chiral limit of the quark-meson model strongly depends on the values for the model parameters. The way how to extrapolate towards the chiral limit is not unique. Thus, the mean-field approximation fails to properly describe the expected critical behavior in the chiral limit at least for the parameter set chosen.

This is remedied in the RG approach and a second-order phase transition, which lies in the expected $O(4)$ universality class, is found in the chiral limit at finite temperature [22, 23]. For finite chemical potential the second-order transition ends in a TCP. For finite quark or pion masses this transition is washed out and becomes a smooth crossover with a critical endpoint. Thus, in the RG framework the relationship and the correlations between the TCP and the various CEP's, obtained by varying the pion mass, can be studied. In addition, the influence of fluctuations on the susceptibilities and the critical region around the CEP can be assessed.

The resulting phase diagrams for the chiral limit and for physical pion masses $M_{\pi} \sim 130 \mathrm{MeV}$ are both shown in Fig. 2. The location of the TCP for our choice of parameters is at $T_{c}^{t} \sim 80$ $\mathrm{MeV}$ and $\mu_{c}^{t} \sim 270 \mathrm{MeV}$. For temperatures below the TCP the phase transition changes initially to a first-order transition. For temperatures below $10 \mathrm{MeV}$ two phase transitions with a second tricritical point emerge [24]. A larger constituent quark mass pushes the location of the first TCP towards the temperature axis and the location of the splitting point of the two phase transition lines 
down towards the $\mu$-axis. All qualitative features of the two transition lines survive, only the area bounded by the two transition lines is reduced for increasing quark masses. Thus, in contrast to the mean-field approximation the RG method yields a TCP in the phase diagram for the chiral limit as expected.

As the pions become massive the TCP turns into a CEP, which lies in the universality class of the three-dimensional Ising model. The location of the CEP for physical pion masses is at $T_{c}^{c} \sim 62$ $\mathrm{MeV}$ and $m_{c}^{c} \sim 313 \mathrm{MeV}$. Compared to recent lattice and other model studies the location of the TCP and consequently of the CEP is at lower temperatures due to the omission of other degrees of freedom in the used quark-meson model. Typical for the RG treatment is the bending of the first-order transition lines for smaller temperatures. But very close to the $\mu$-axis the slope of the first-order boundary $d T / d \mu$ tends to infinity similar to the mean-field phase diagram and is also in agreement with the Clausius-Clapeyron relation [25]. In the chiral limit below the splitting point the right second-order transition line turns into an crossover for finite quark masses which is not visible in Fig. 2. Analogously, the second TCP should turn into a critical point whose remnants can be seen in the order parameter and meson masses [24].

The results of a recalculation of the contour plot around the CEP in the framework of the RG approach is also shown in Fig. 1. The critical region is again elongated in the direction of the firstorder transition line, but it is now much more compressed. While the interval of the critical region in the temperature direction is comparable with the one obtained in the mean-field approximation, the effect in the chemical potential direction is dramatic. In the RG calculation the interval is shrunken by almost one order of magnitude, despite the fact that the corresponding critical exponents are quite similar. For example, at the CEP the susceptibility diverges with the critical exponent $\varepsilon \sim 0.74$ which is consistent with the one of the expected 3D Ising universality class $\varepsilon=0.78$. Thus, as a consequence of fluctuations, the size of the critical region around the CEP is substantially reduced as compared to the mean-field calculation. This may also have consequences for the experimental localization of the CEP in the phase diagram since it further complicates its detection through event-by-event fluctuations.

\section{The quark-meson model with Polyakov-loop dynamics}

Despite the success of the RG approach in predicting the expected critical behavior of the thermodynamics in the quark-meson model, explicit gluonic degrees of freedom, which are known to play an important role in the thermodynamics of QCD and are associated with confinement aspects are missing in this model. One possibility to incorporate such effects is the coupling of the quark-meson model to the Polyakov loop. This results in an coupled effective Polyakov-quarkmeson (PQM) model with an interaction potential between quarks, mesons and the Polyakov loop variables $\phi, \bar{\phi}$. The PQM model includes the chiral aspects of QCD as well as certain aspects of confinement.

The order parameter $\langle\phi\rangle$, and respectively $\langle\bar{\phi}\rangle$, vanishes in the confined phase where the free energy of a single heavy quark, respectively antiquark, diverges and is finite in the deconfined phase. In the presence of dynamical quarks, the free energy of a quark-antiquark pair does not diverge anymore, and the order parameter is always non-vanishing. For finite quark chemical potential the free energies of quarks and antiquarks are different. Since $\langle\phi\rangle$ is related to the free 
energy of quarks and the hermitian conjugate $\langle\bar{\phi}\rangle$ to that of antiquarks, their modulus differs in general.

In pure Yang-Mills theory the mean value $\langle\phi\rangle,\langle\bar{\phi}\rangle$ are given by the minima of the effective Polyakov-loop potential $U(\phi, \bar{\phi})$. It can be constructed from lattice data for the expectation values [26] or from a RG calculation [27]. Here, a polynomial expansion in $\langle\phi\rangle,\langle\bar{\phi}\rangle$ up to quartic terms is used. The expansion coefficients are fixed to reproduce thermodynamic lattice results for the pure Yang-Mills sector. This potential has a first-order phase transition at the critical temperature $T_{0}=270 \mathrm{MeV}$. In the presence of dynamical quarks, the running gauge coupling is changed due to fermionic contributions. In our approximation to the Polyakov-loop potential this only leads to a modification of the first expansion coefficient $b_{2}$ in front of the quadratic fields. The size of this effect can be estimated within perturbation theory. At zero temperature it leads to an $N_{f}$-dependent decrease of $\Lambda_{\mathrm{QCD}}$, which translates into an $N_{f}$-dependent decrease of the critical temperature $T_{0}$ at finite temperature. Table 1 shows the results for the $N_{f}$-dependent critical temperature $T_{0}$ in the Polyakov-loop potential for massless flavors. Massive flavors lead to a suppression factor in the $\beta$-function of QCD which modifies $T_{0}$ further. E.g. for $2+1$ flavors with a current strange quark mass $m_{s} \sim 150 \mathrm{MeV}$ a $T_{0}(2+1) \sim 187 \mathrm{MeV}$ is obtained.

\begin{tabular}{c||c|c|c|c|c}
$N_{f}$ & 0 & 1 & 2 & $2+1$ & 3 \\
\hline \hline$T_{0}[\mathrm{MeV}]$ & 270 & 240 & 208 & 187 & 178 \\
\hline
\end{tabular}

Table 1: The critical Polyakov-loop temperature $T_{0}$ for $N_{f}$ massless flavors.

A second step implements a $\mu$-dependent running coupling in the $b_{2}$ coefficient, analogous to the $N_{f}$-dependence discussed above. One can argue that this is a minimal necessary generalization because without a $\mu$-dependent $b_{2}$ coefficient the confinement-deconfinement phase transition has a higher critical temperature than the chiral phase transition at vanishing chemical potential. But this is an unphysical scenario because QCD with dynamical massless quarks in the chirally restored phase cannot be confining since the string breaking scale would be zero.

As for the $N_{f}$-dependence one can resort to perturbative estimates, by allowing for an additional $\mu$-dependent term in the one-loop coefficient of the QCD $\beta$-function, which can be motivated by using HTL/HDL results. This additional coefficient can be fixed such that the chiral transition temperature and the confinement-deconfinement transition agree at some arbitrary non-vanishing $\mu$. Interestingly, it turns out that then the transition temperatures agree for all values of $\mu$. This $\mu$-dependence in the $\beta$-function then leads to a $T_{0}$ with an additional $\mu$-dependence, such that $T_{0} \rightarrow T_{0}\left(\mu, N_{f}\right)$. Of course, these novel modifications should be viewed as a rough estimate of the $\mu$-dependence of $T_{0}$. For a more quantitative analysis the non-perturbative running of the coupling in the presence of finite temperature and quark density has to be considered. This can be incorporated in a self-consistent RG-setting.

The phase structure of the PQM model is determined by the behavior of the order parameters $\langle\sigma\rangle,\langle\phi\rangle$ and $\langle\bar{\phi}\rangle$ and the grand canonical potential as a function of temperature and quark chemical potential. The phase diagram in the $(T, \mu)$-plane resulting from the two flavor PQM models in mean-field approximation is shown in Fig. 3 (upper lines). The bottom lines in this figure display the phase diagram of the pure quark-meson model without the Polyakov loop dynamic. 


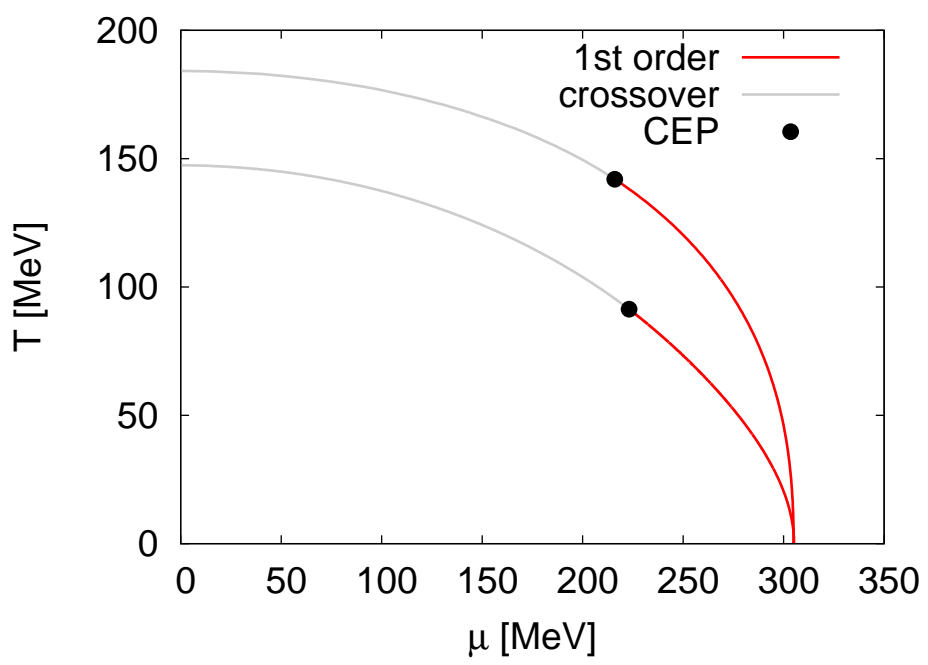

Figure 3: Phase diagram of the quark-meson model with Polyakov loop (upper lines) and without Polyakov loop dynamics (lower lines) in mean-field approximation.

With the Polyakov loop modifications a chiral crossover temperature $T_{c} \sim 184 \mathrm{MeV}$ at $\mu=0$ is found with an uncertainty of $\sim 14 \mathrm{MeV}$ originating in the error estimate $\pm 30 \mathrm{MeV}$ for $T_{0}$. As an example for the estimate of the error, the two-loop running of the gauge coupling leads to a $T_{0} \sim 192 \mathrm{MeV}$ and hence a $T_{c} \sim 177 \mathrm{MeV}$. In the presence of dynamical quarks the Polyakov loop shows also a crossover at the same pseudocritical temperature which can be read off from the peak position of $\partial\langle\bar{q} q\rangle / \partial T$ and $\partial\langle\phi\rangle / \partial T$.

Recently, a recalculation of the transition temperature on different lattices for two light and one heavier quark mass close to their physical values yields inconsistent results: On the one hand using the Sommer parameter $r_{0}$ for the continuum extrapolation a $T_{c}=192 \pm 7 \mathrm{MeV}$ is found [28] and on the other hand in another analysis with four different sets of lattice sizes $N_{\tau}=4,6,8$ and $10 \mathrm{a}$ $T_{c}=151 \pm 3 \mathrm{MeV}$ is obtained [29]. Within an Functional RG approach a critical value of $T_{c}=172$ $\mathrm{MeV}$ [30] is achieved which again agrees with a $T_{c}=173 \pm 8 \mathrm{MeV}$ obtained in former two-flavor lattice simulations with improved staggered fermions extrapolated to the chiral limit [31]. Using the same parameters for the quark-meson model without the Polyakov-loop modifications a crossover temperature of $T_{c} \sim 150 \mathrm{MeV}$ emerges [10, 24]. This temperature gap calls for refined studies both on the lattice as well as analytical methods to resolve this discrepancy.

With and without the Polyakov loop modifications the phase diagram features a critical endpoint (CEP), where the line of first-order transitions terminates in a second-order transition. Lattice simulations are not conclusive concerning the existence and location of the CEP. There are indications from lattice simulations at small chemical potentials that deconfinement and chiral symmetry restoration appear along the same critical line in the phase diagram. For the PQM model with an $\mu$-independent $T_{0}$ the coincidence of deconfinement and chiral transition at $\mu=0$ disappears for finite $\mu$. The deconfinement temperature is larger than the corresponding chiral transition temperature. This is an unphysical scenario because the deconfinement temperature should be smaller or equal to the chiral transition temperature. Contrarily, with the $\mu$-dependent $T_{0}$ coinciding transition lines for the entire phase diagram within an accuracy of $\pm 5 \mathrm{MeV}$ are found. 


\section{Summary}

The phase diagram of hadronic matter is analyzed in the two-flavor quark-meson model by means of a Wilsonian RG approach. This model captures essential features of QCD such as chiral symmetry breaking in the vacuum and can therefore yield valuable insight into the critical behavior associated with chiral symmetry. Of special importance is the emergence of a CEP and the size of the critical region around the CEP in connection with fluctuation signals in heavy-ion collisions. Most studies of this issue have been performed in the mean-field approximation which neglects thermal and quantum fluctuations. These can be assessed in the RG approach which is able to correctly predict critical exponents in the vicinity of critical points of the phase diagram.

In a mean-field calculation no TCP is found for the chosen parameter set while the RG predicts its existence as is expected from universality arguments. Because of the Gaussian fixed point structure at the TCP mean-field exponents are expected what we also could verify. When effects of finite current quark masses (or equivalently finite pion masses) are included, a CEP emerges in both the mean-field and RG calculation. By analyzing the scalar- and quark number susceptibilities with the RG approach we found nontrivial critical exponents which are consistent with the expected 3D Ising universality class. As a consequence of fluctuations the size of the critical region around the CEP is substantially reduced as compared to the mean-field results. This is particularly true in the $\mu$-direction.

One of the truncations of the quark-meson model is the lack of explicit gluonic degrees of freedom. This is addressed by the introduction of the PQM model that includes certain aspects of gluon dynamics via the Polyakov loop and represents a minimal synthesis of the two basic principles of QCD at low temperatures: spontaneous chiral symmetry breaking and confinement.

A limited set of input parameters is adjusted to reproduce lattice QCD results in the pure gauge sector and pion properties in the hadron sector. Then the PQM model correctly describes the step from the first-order deconfinement transition observed in pure-gauge lattice QCD with a $T_{c} \sim 270$ $\mathrm{MeV}$ to the crossover phenomenon with a pseudocritical $T_{c}$ around $200 \mathrm{MeV}$ when two light quark flavors are added. The non-trivial result is that the crossovers for chiral symmetry restoration and deconfinement almost coincide at small $\mu$ similar to lattice simulations. Via RG arguments it is possible to estimate an $N_{f^{-}}$and $\mu$-dependence in the parameters of the Polyakov loop potential: the critical temperature of the Polyakov loop model decreases with increasing $N_{f}$ and $\mu$. These modifications yield coinciding peaks in the temperature derivative of the Polyakov loop expectation value and the chiral condensate at $\mu=0$. Interestingly, this coincidence of the deconfinement and chiral symmetry restoration persists at finite $\mu$. These findings provide a promising starting point for a functional RG study in the PQM model, and further extensions towards full QCD.

\section{Acknowledgment}

The work presented here was done in collaboration with Jochen Wambach. The study on the PQM model was done also in collaboration with Jan M. Pawlowski. The author is grateful to the Technical University of Darmstadt and GSI for the hospitality. This work is supported by the BMBF Grant No. 06DA116. 


\section{References}

[1] M. Asakawa and K. Yazaki, Nucl. Phys. A 504, 668 (1989).

[2] A. Barducci, R. Casalbuoni, S. De Curtis, R. Gatto and G. Pettini, Phys. Lett. B 231, 463 (1989).

[3] J. Berges and K. Rajagopal, Nucl. Phys. B 538, 215 (1999).

[4] R.D. Pisarski and F. Wilczek, Phys. Rev. D 29, 338 (1984).

[5] Z. Fodor and S.D. Katz, Phys. Lett. B 534, 87 (2002).

[6] P. de Forcrand and O. Philipsen, Nucl. Phys. B 642, 290 (2002).

[7] C.R. Allton et al., Phys. Rev. D 68, 014507 (2003).

[8] D.F. Litim and J.M. Pawlowski, arXiv:hep-th/9901063; J. Polonyi, Central Eur. J. Phys. 1, 1 (2003); J. Berges, N. Tetradis and C. Wetterich, Phys. Rept. 363, 223 (2002); J.M. Pawlowski, arXiv:hep-th/0512261v2; H. Gies, arXiv:hep-ph/0611146.

[9] B.-J. Schaefer and J. Wambach, arXiv:hep-ph/0611191.

[10] B.-J. Schaefer and J. Wambach, Phys. Rev. D75, 085015 (2007).

[11] D.-U. Jungnickel and C. Wetterich, Phys. Rev. D 53, 5142 (1996).

[12] B.-J. Schaefer and H.-J. Pirner, Nucl. Phys. A660, 439 (1999).

[13] K. Fukushima, Ann. Phys. 304, 72 (2003); P.N. Meisinger, T.R. Miller and M.C. Ogilvie, Nucl. Phys. Proc. Suppl. 119511 (2003).

[14] C. Ratti, M.A. Thaler and W. Weise, Phys. Rev. D73, 014019 (2006).

[15] E. Megias, E. Ruiz Arriola and L.L. Salcedo, Phys. Rev. D74 , 065005 (2006).

[16] C. Sasaki, B. Friman and K. Redlich, arXiv:hep-ph/0611147.

[17] B.-J. Schaefer, J.M. Pawlowski and J. Wambach, arXiv:0704.3234 [hep-ph], to appear in Phys. Rev. D.

[18] Y. Hatta and T. Ikeda, Phys. Rev. D 67, 014028 (2003).

[19] O. Scavenius, A. Mocsy, I.N. Mishustin and D.H. Rischke, Phys. Rev. C 64045202 (2001).

[20] C. Sasaki, B. Friman and K. Redlich, arXiv:hep-ph/0 702254; see also contribution to these proceedings.

[21] H. Fujii and M. Ohtani, Phys. Rev. D 70, 014016 (2004).

[22] J. Berges, D.-U. Jungnickel and C. Wetterich, Int. J. Mod. Phys. A 18, 3189 (2003).

[23] N. Tetradis, Nucl. Phys. A 726, 93 (2003).

[24] B.-J. Schaefer and J. Wambach, Nucl. Phys. A757, 479 (2005).

[25] A. Aigner and B.-J. Schaefer, in preparation.

[26] R.D. Pisarski, Phys. Rev D 62, 111501 (2000).

[27] J. Braun, H. Gies and J.M. Pawlowski, arXiv:0708.2413 [hep-th] .

[28] M. Cheng et al., Phys. Rev. D 74, 054507 (2006).

[29] Y. Aoki, Z. Fodor, S.D. Katz and K.K. Szabo, Phys. Lett. B 643, 46 (2006).

[30] J. Braun and H. Gies, JHEP 06, 024 (2006); Phys. Lett. B 645, 53 (2007).

[31] F. Karsch, Lect. Notes Phys. 583, 209 (2002). 